\title{
Images of the Quenching of a Flame by a Vortex-To Quantify Regimes of Turbulent Combustion
}

\author{
WILLIAM L. ROBERTS and JAMES F. DRISCOLL \\ Department of Aerospace Engineering, University of Michigan, Ann Arbor, MI 48109
}

MICHAEL C. DRAKE

General Motors Research Laboratories, Warren, MI 48090

LARRY P. GOSS

Systems Research Laboratories, Dayton, OH 45440

\begin{abstract}
A laminar toroidal vortex is interacted with a laminar premixed flame in order to isolate and to visualize some of the fundamental physics of turbulent combustion. Localized quenching of the flame was observed using planar laser-induced fluorescence imaging of superequilibrium $\mathrm{OH}$ molecules in the counterflow flamefront region near the vortex leading edge. A quenching limit curve was measured as a function of vortex size and strength. In the second part of the study, the measurements are combined with concepts proposed by Poinsot, Veynante, and Candel in order to infer the thin flame limit, namely, the onset of distributed reactions, on a classical premixed turbulent combustion regime diagram. The measured thin flame limit indicates when laminar flamelet theories become invalid, since quenching allows hot products and reactants to coexist. Results are compared with the Klimov-Williams criterion. Vortex core diameters were as small as the flame thickness in some cases. The main conclusion is that small vortices are less effective at quenching a flame than was previously believed; therefore the inferred regime within which thin flame theories are valid extends to a turbulence intensity that is more than an order of magnitude larger than that which was previously predicted. Results also indicate that micromixing models, which assume that the smallest eddies exert the largest strain on a flame, are not realistic. Measured trends are in agreement with direct numerical simulations of Poinsot et al., but absolute values differ. The measured vortex Karlovitz number that is required to quench a flame is not constant but decreases by a factor of four as vortex size increases from one to five flame thicknesses. Thin-film pyrometry was used to quantify the radiative heat losses; quenching occurs when the products cool to approximately $1300 \mathrm{~K}$, which is in agreement with stretched laminar flame calculations that include detailed chemistry. The quenching Karlovitz number for propane-air flames differs from that of methane-air flames, indicating the importance of detailed chemistry and transport properties. Flame curvature was observed to cause enhancement (or reduction) of the local reaction rate, depending on the Lewis number, in a manner that is consistent with stretched flame theory.
\end{abstract}

\section{INTRODUCTION}

The present work is an investigation of the vortex strength that is required for a single eddy in a turbulent flow to locally quench a premixed flame, and thereby alter the flame structure. This quenching condition is denoted the thin flame limit. When a flame is locally quenched, the reactants can coexist with hot products prior to chemical reaction, which can result in a distributed reaction. This coexistence of reactants and products violates the assumptions made in thin flame theories.
Therefore it is useful to quantify the thin flame limit in order to determine the range over which thin, strained flamelet models are valid.

The classical regime diagram for turbulent premixed flames appears in Ref. 1 and is discussed below. The predicted thin flame limit is reached when the turbulence intensity is sufficient to cause the nondimensional stretch rate (Karlovitz number) that is based on the Taylor microscale $\left(u_{\mathrm{rms}}^{\prime} / \Lambda\right) /\left(S_{L} / \delta\right)$ to equal unity. This prediction has been denoted the Klimov-Williams criterion [2]; the Taylor microscale $(\Lambda)$ is assumed to be the appropriate 
scale because it is the characteristic scale of the strain rate in a nonreacting turbulent flow [3]. The rms velocity fluctuation is $u_{\mathrm{rms}}^{\prime}, S_{L}$ is the laminar burning velocity, and $\delta$ is the laminar flame thickness. Previously there have been no measurements available to assess how vortices quench flames or to determine if quenching occurs at a constant Karlovitz number. Numerical results have been reported by Poinsot et al. [4, 5], which predict when a single vortex can quench a flame; Poinsot et al. also show how to relate single vortex simulations to the regime diagram for turbulent combustion. However, none of the simulations to date can yield quantitative values because the simulations include unrealistic heat loss terms and unrealistic one-step chemistry. Flame quenching is especially sensitive to both assumptions, so quantitative results must come from experiment.

A second research issue is whether or not small vortices play a dominant role in the process of flame wrinkling and flame quenching. Micromixing models [6] assume that small vortices having a core diameter $\left(d_{c}\right)$ near the Kolmogorov or the Taylor scales play a dominant role because in a nonreacting flow the strain rate $U_{\theta} / d_{c}$ that is associated with eddies of diameter $d_{c}$ scales as $d_{c}^{-2 / 3}$ and thus is largest for small eddies. That is, eddy rotational velocity $U_{\theta}$ is assumed to be unaffected by the flame and to scale as $d_{c}{ }^{1 / 3}$, according to turbulent energy cascade concepts [3]. In contrast, simulations by Poinsot et al. $[4,5]$ and the present measurements show that small eddies do not strain out the flame because they are destroyed by viscous forces. The importance of this finding is that it means that direct numerical simulations of turbulent flames need not attempt to resolve all of the small scales of turbulence. However, measurements such as those herein are needed to determine the range of small scales that can be ignored.

The approach taken has been to interact a single laminar, toroidal vortex with a laminar flame. The smallest vortex used had a toroid diameter of $1.27 \mathrm{~cm}$ and a vortex core diameter $\left(d_{c}\right)$ of $0.25 \mathrm{~cm}$. It was decided to operate near the lean flammability limit in order to achieve a large value of the thermal thickness of the flame $(\delta)$ of $0.26 \mathrm{~cm}$ so that values of $d_{c} / \delta$ as small as unity could be achieved. Our previous studies $[7,8]$ provided the first conclusive experimental evidence that a premixed flame can be quenched by a vortex. Only one vortex size was considered in that work, so the present work utilizes a range of vortex diameters, vortex strengths, and several different values of Lewis number. A toroidal vortex was chosen because two-dimensional linear vortices tend to be unstable, especially at the large rotational velocities desired. Another requirement was that naturally-occurring radiative heat losses must be sufficient to prevent the hot products from immediately reigniting the flame; in addition, the vortex must have sufficient residence time in the flame to cause quenching.

Figure 1 illustrates that the physics of the present flame quenching process is similar to that of a counterflow flame that is produced between a stream of cold reactants and an opposing stream of hot products. The counterflow flame problem has been solved by several researchers, including Darabiha et al. [9, 10]. Near the vortex leading edge in Fig. 1 there exists a stagnation point; cold reactants approach the stagnation point from above, and due to the vortex downward motion, hot products approach the stagnation point from below. It is relatively difficult to quench a counterflow flame without sufficient heat loss because hot products always remain in contact with reactants. However, Darabiha et al. [9] show that sufficient strain forces the flame to move across the stagnation point. For such conditions, incomplete reaction occurs, since most of the reactants remain on the reactant side of the

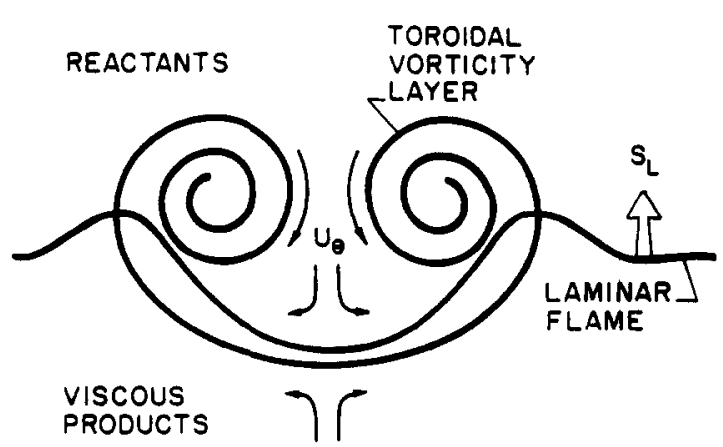

Fig. 1. Schematic showing how the flame near the vortex leading edge is similar to a counterflow flame. 
stagnation point. Only those reactants that can diffuse across the opposing convection field can be consumed at the flame. The maximum temperature and the rate of reactants consumed per unit volume of the reaction zone do not decrease due to strain in Darabiha's solution. Instead the increasing strain reduces the reaction zone thickness to zero, the reactants bypass the flame, and the flame is quenched.

Heat losses are important in nearly all flame quenching experiments and substantial heat losses had to be added to the numerical simulations of Poinsot et al. $[4,5]$ in order to observe quenching. Therefore, the present strategy was not to attempt to eliminate heat losses but to quantify the naturally occurring radiation losses in the experiment. Measurements were made of the rate at which gas temperature decreases with distance behind the flame due to radiation; the measured temperature profiles could be used to insure that numerical simulations properly match the realistic radiative losses typical of the present experiment.

The counterrotating vortex pair chosen for this study, as shown in Fig. 1, is not the only possible arrangement of vortices upstream of a turbulent flame. However, this arrangement, which exerts extensive strain on the flame, was chosen because it is the most probable quenching mechanism, as demonstrated by direct simulations $[11,12]$. If one considers any vortex in the reactants ahead of the flame and one proceeds along the flame until an adjacent vortex is encountered, the two vortices can be (a) corotating, (b) counterrotating with the reactants between the vortices directed away from the flame, thereby exerting compressive strain on the flame, and (c) counterrotating with the reactants between the vortices directed towards the flame, thereby inducing extensive strain on the flame. The compressive strain case (b) is least probable because the flame will propagate most rapidly over this vortex pair since reactants between the vortices are moving in the direction of flame propagation. The extensive strain case (c) is most probable because the flame spends a significantly longer residence time attempting to propagate over a flowfield that is directed against the propaga- tion direction. Wu and Driscoll [13] have quan. tified the different residence times associatec with the above three cases.

\section{EXPERIMENTAL ARRANGEMENT}

The experiment is shown schematically in Fig 2 and is discussed in detail in Refs. 7 and 8 . It consists of a rectangular flame tube having dimensions of $11.4 \mathrm{~cm}$ by $11.4 \mathrm{~cm}$ by $61 \mathrm{~cm}$. A single toroidal laminar vortex is formed by using a loudspeaker to impulsively force fluid downward through a sharp edged orifice. The orifice diameter is denoted $\mathrm{D}$ and was varied between 1.27 and $5.1 \mathrm{~cm}$. Laser velocimetry [8] was used to measure the vortex velocity profiles. It was found that the diameter of the toroidal vortex ring is equal to the orifice diameter $D$, as is shown in Fig. 1, to within $10 \%$ accuracy. The diameter of the vortex core is denoted $d_{c}$; the core is defined as the region of solid body rotation. The core diameter $d_{c}$ was found to equal $0.2 D$ at the measurement loca-

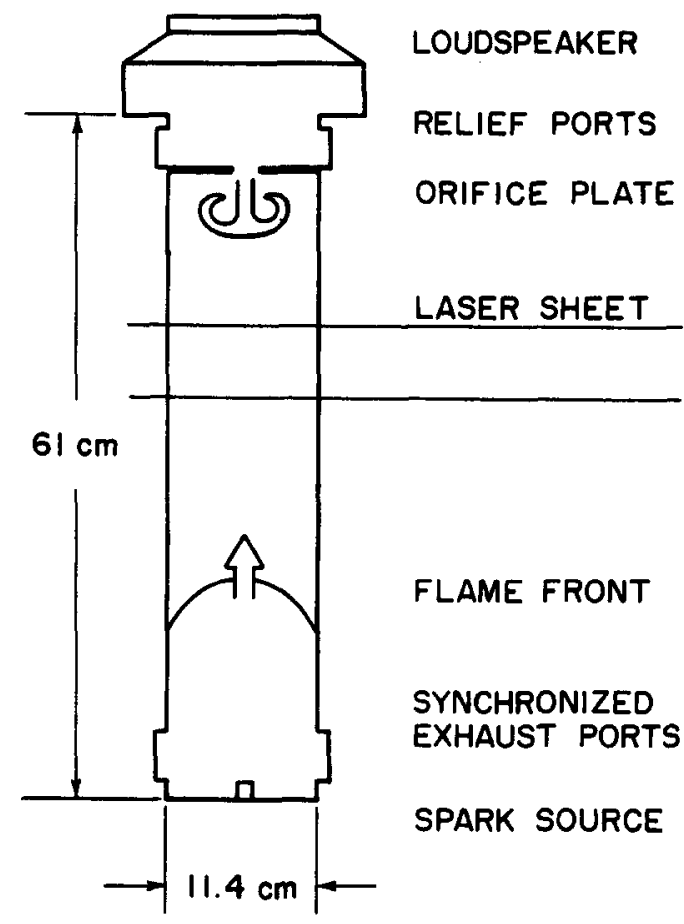

Fig. 2. Schematic of the flame-vortex interaction apparatus. 
tion for the vortices considered. At other locations downstream, the vortex diameter will increase due to entrainment of fluid into the vortex. The quantity $U_{\theta}$ is the maximum rotational velocity within the vortex, which occurs at a distance of $d_{c} / 2$ from the center of the core, which is expected for a Rankine vortex.

A homogeneous fuel-air mixture flows through the chamber before the chamber is sealed and the charge is ignited, causing a laminar flame to propagate upward. The equivalence ratio was varied between 0.50 and 0.70 and three fuels (methane, propane and ethane) were used. Tables 1 and 2 list the laminar burning velocities $\left(S_{L}\right)$ and thermal thicknesses of the flames studied. $S_{L}$ was determined by subtracting the gas velocity ahead of the flame (which was measured using laser velocimetry to be $34 \mathrm{~cm} / \mathrm{s}$ for methane, $\phi=0.6$ ), from the flame speed in lab coordinates, which was determined to be $42 \mathrm{~cm} / \mathrm{s}$ for methane, $\phi=0.6$, using motion picture images of Mie scattering. The resulting values of $S_{L}$ are in agreement with those reported in Refs. 18-20.

The thermal thickness of the flame $(\delta)$ is defined as the temperature difference across the flame divided by the maximum temperature gradient. Four previous studies [14-17] report temperature profiles that indicate that $\delta$ equals $7.4 \alpha / S_{L}$ for lean hydrocarbon flames to within an accuracy of $10 \% ; \alpha$ is the thermal diffusivity of the cold reactants. Therefore $\delta$ was determined from this relation. It is noted
TABLE 2

Properties of the Flames Studied

\begin{tabular}{ccrccc}
\hline Fuel & $\phi$ & $\begin{array}{c}S_{L} \\
(\mathrm{~cm} / \mathrm{s})\end{array}$ & $\begin{array}{c}\delta^{a} \\
(\mathrm{~cm})\end{array}$ & $\begin{array}{c}T_{\text {max,meas }}{ }^{b} \\
(\mathrm{~K})\end{array}$ & $\begin{array}{c}T_{\mathrm{ad}}{ }^{c} \\
(\mathrm{~K})\end{array}$ \\
\hline Methane & 0.55 & 6.5 & 0.26 & 1525 & 1573 \\
Methane & 0.60 & 8.0 & 0.20 & 1605 & 1664 \\
Methane & 0.70 & 12.0 & 0.14 & 1810 & 1838 \\
Propane & 0.50 & 8.0 & 0.20 & 1479 & 1507 \\
Propane & 0.60 & 14.0 & 0.12 & 1688 & 1700 \\
\hline
\end{tabular}

${ }^{a}$ The thermal thickness of the flame $\delta$ is $7.4 \alpha / S_{L}$ as discussed in the text.

\footnotetext{
${ }^{b} T_{\text {max, meas }}$ is maximum temperature measured with thin filament pyrometry.

${ }^{c} T_{\text {ad }}$ is adiabatic flame temperature calculated by the NASA CEC-72 equilibrium code.
}

that others $[4,5]$ use a somewhat different definition of $\delta$. Two conflicting requirements that had to be met were that the vortex Karlovitz number $\left(U_{\theta} / S_{L}\right) /\left(d_{c} / \delta\right)$ must be of order unity for quenching to occur, while the Reynolds number $U_{\theta} D / \nu$ must be less than 1200 to maintain a laminar vortex [8]. It follows that $S_{L} D$ must be less than $255 \nu$, so it is advantageous to operate near the lean flammability limit to minimize the burning velocity $S_{L}$.

The maximum rotational velocity within the vortex was defined as $U_{\theta}$ and was found to increase linearly with $\Delta V^{2} /\left(t_{r} D^{5}\right)$, [8], where $\Delta V$ is the volume displaced by the loudspeaker and $t_{r}$ is the time duration of loudspeaker motion. This relation is derived by equating the circulation of the vortex, which is propor-

TABLE 1

Parameters Associated with the Images Shown in Figs. 3-8

\begin{tabular}{clcccccccc}
\hline Fig. & Fuel & $\phi$ & $d_{c} / \delta$ & $\mathrm{Ka}_{v}$ & $\begin{array}{c}d_{c} \\
(\mathrm{~cm})\end{array}$ & $\begin{array}{c}U_{\theta} \\
(\mathrm{cm} / \mathrm{s})\end{array}$ & $U_{\theta} / S_{L}$ & $\begin{array}{c}K \\
\left(\mathrm{~s}^{-1}\right)\end{array}$ & $\begin{array}{c}T_{Q} \\
(\mathrm{~K})\end{array}$ \\
\hline 3a & methane & 0.55 & 1.0 & 14.0 & 0.25 & 91 & 14.0 & 365 & 1300 \\
3b & methane & 0.55 & 2.0 & 6.2 & 0.51 & 80 & 12.3 & 157 & 1300 \\
3c & methane & 0.55 & 3.1 & 4.5 & 0.76 & 90 & 13.8 & 119 & 1300 \\
7a & methane & 0.55 & 2.0 & 6.3 & 0.51 & 82 & 12.5 & 160 & 1300 \\
7b & propane & 0.60 & 4.6 & 2.0 & 0.51 & 130 & 9.3 & 255 & no quen. \\
$7 \mathrm{c}$ & propane & 0.50 & 5.3 & 3.8 & 1.02 & 160 & 20.0 & 157 & no quen. \\
$7 \mathrm{~d}$ & ethane & 0.56 & 5.1 & 3.9 & 1.02 & 160 & 20.0 & 157 & no quen. \\
8a & propane & 0.50 & 2.0 & 6.0 & 0.38 & 96 & 12.0 & 252 & no quen. \\
8b & propane & 0.50 & 4.0 & 2.8 & 0.76 & 90 & 11.3 & 118 & no quen. \\
8c & ethane & 0.55 & 3.8 & 3.0 & 0.76 & 90 & 11.3 & 118 & no quen. \\
8d & methane & 0.56 & 2.0 & 6.2 & 0.51 & 80 & 12.3 & 157 & no quen. \\
\hline
\end{tabular}

Strain rate $K=U_{\theta} / d_{c} ;$ Core diameter $d_{c}=0.2 \mathrm{D} ; D=$ orifice diameter; Vortex Karlovitz number $\mathrm{Ka}_{v}=\left(U_{\theta} / S_{L} /\right.$ $\left(d_{c} / \delta\right) ; \delta=$ flame thickness $=7.4 \alpha / S_{L} ; T_{Q}=$ temperature of products when quenching occurs. 
tional to $U_{\theta} D$, to the circulation within the segment of the boundary layer that exits the orifice during time $t_{r}$. The boundary layer circulation is proportional to the product of the boundary layer vorticity (which scales with $U / \delta$, where $U$ is the orifice velocity and $\delta$ is the boundary layer height) and the boundary layer area, which scales with $\delta U t_{r} . U$ is eliminated by equating it to $\left(\Delta V / t_{r}\right) /\left(\pi D^{2} / 4\right)$ in order to satisfy conservation of mass.

Flame quenching was detected using a planar laser induced $\mathrm{OH}$ fluorescence system. A Nd:YAG pumped dye laser with a frequency doubled output of $2 \mathrm{~mJ}$ at $282.7 \mathrm{~nm}$ was tuned to the $Q_{1}(5)$ line of the $(1,0)$ band of the $X^{2} \Pi-A^{2} \Sigma^{+}$transition of the $\mathrm{OH}$ molecule as described by Drake and Pitz [21]. The laser light sheet is $5.1 \mathrm{~cm}$ in height and less than 200 $\mu \mathrm{m}$ thick at the chamber centerline. The laser frequency was tuned after every ten runs by optimizing the $\mathrm{OH}$ laser induced fluorescence signal from a Bunsen burner. Fluorescence was collected in the wavelength range from 300 to $390 \mathrm{~nm}$ using a WG-305 cutoff filter and a UG-11 glass filter and was collected by an $f$ 2.8 UV Nikkor lens which has a $100-\mathrm{mm}$ focal length. Images were recorded by a Princeton Instruments ICCD-576 intensified, cooled CCD array camera that has a $576 \times 384$ pixel array. Each pixel was sampled with 14-bit resolution and stored on an IBM compatible $386 / 33-\mathrm{MHz}$

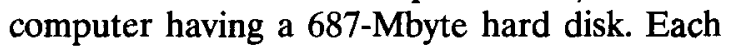
image was corrected for laser sheet intensity variations by normalizing by an image of the Rayleigh scattering from room temperature air.

A Thin Filament Pyrometer (TFP) was employed to measure the time history of temperature on the centerline in order to quantify heat losses from the product gases. The arrangement was similar to that reported by Goss et al. [22]. The concept is that the infrared greybody emission from a very thin filament is proportional to the fourth power of the filament temperature and thus provides a sensitive measure of the gas temperature in the range $700-2400 \mathrm{~K}$. A time response of $0.3 \mathrm{~ms}$ was calculated by using the standard relation for the time response of a thermocouple. A 15- $\mu \mathrm{m}$-diameter, 15 -cm-long SiC filament extended horizontally across the chamber and was attached to the sidewalls. A 1-mm section of the filament was focused onto a InGaA: infrared detector using a $10-\mathrm{cm}$ focal lengtt lens and optical filters with a bandpass 0 : $0.8-1.8 \mu \mathrm{m}$. Gas temperature differs from the filament temperature by only $1 \%-5 \%$; this difference was determined by using the stan. dard relation that is used to correct thermo. couples for radiation and conduction losses [22]. The TFP output signal was calibrated by placing a stoichiometric propane-air grid-stabilized flame directly under the filament.

\section{RESULTS}

\section{OH Images of the Quenching Process}

Figure 3 illustrates some images of the quenching of a methane-air flame by small, medium and large vortices. The properties of the vortices and flames are listed in Tables 1 and 2. The flame in Fig 3a appears as a red line, where the red color indicates locations where the $\mathrm{OH}$ fluorescence intensity is in the range $50 \%-100 \%$ of the maximum intensity. Note that the product gases, which are below the flame in Figure 3a, appear black, indicating that the fluorescence from the equilibrium $\mathrm{OH}$ in the products is less than $10 \%$ of the fluorescence from the superequilibrium $\mathrm{OH}$ in the flamefront. Drake and Blint [23] have shown that the ratio of superequilibrium $\mathrm{OH}$ to equilibrium $\mathrm{OH}$ concentrations typically exceeds ten for very lean (or rich) flame conditions but will be less than two for adiabatic stoichiometric flames. Therefore, since the present flames are near the lean limit, the $\mathrm{OH}$ fluorescence intensity serves as a marker of the leading edge and the trailing edge of the flame.

It is observed that the pocket of reactants formed by the small vortex does not roll up but retains an oval shape in Fig. 3a, image iii. The following image (iv) shows that the pocket of reactants quenches near the vortex leading edge, where the image appears black and the superequilibrium $\mathrm{OH}$ intensity is measured to be less than $2 \%$ of the maximum intensity in an undisturbed flame. Numerical simulations by Poinsot et al. $[4,5]$ for $d_{c} / \delta$ of approximately unity also show that the pocket fails to roll up and it quenches at the leading edge. The quenching of flames about pockets of re- 

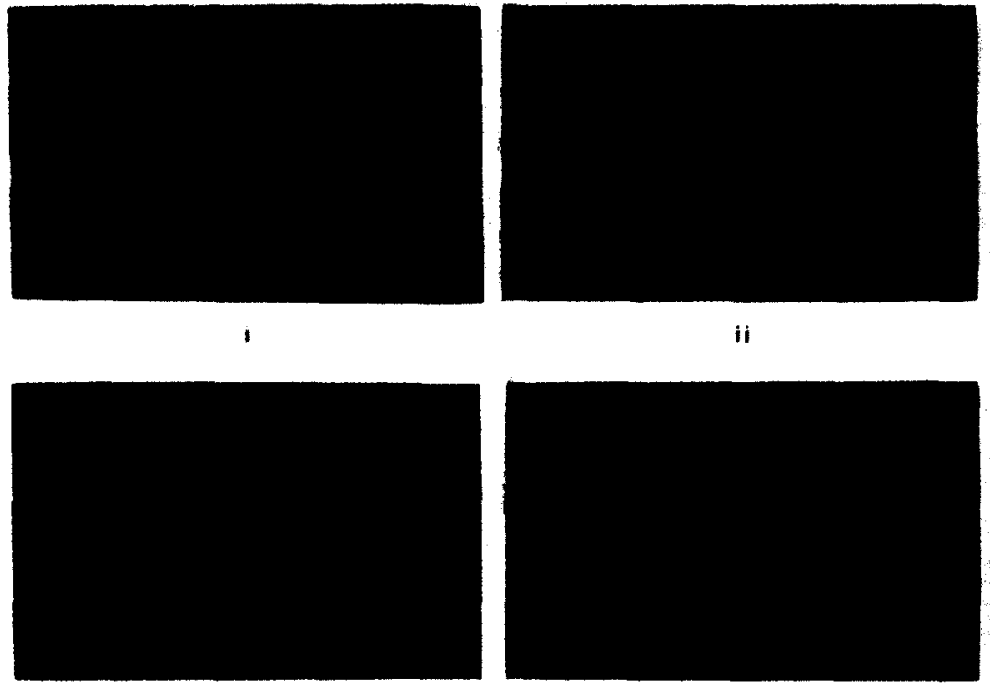

iii

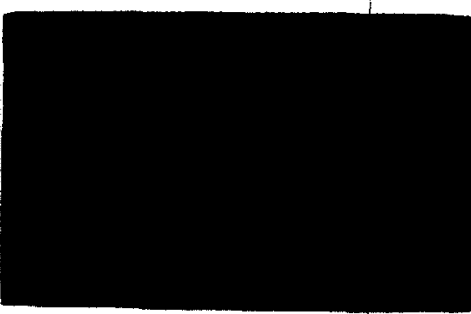

$v \mathbf{i}$

(a) SMALL VORTEX, $d_{d} \delta=1.0, \mathrm{~K}_{v}=14.0$, METHANE
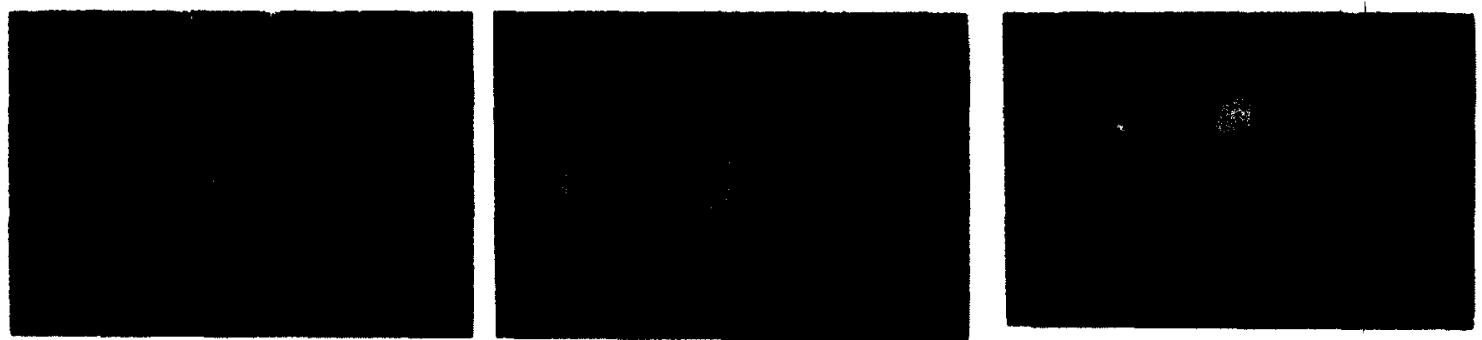

(b) MEDIUM VORTEX, $d \delta \delta=2.0, K a_{v}=6.2$, METHANE

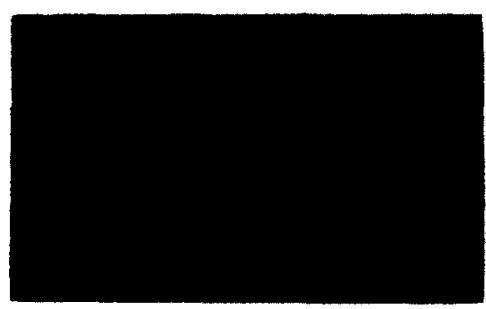

i

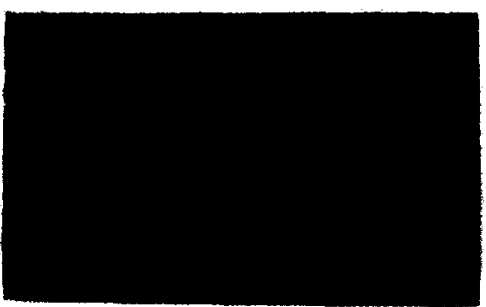

ii

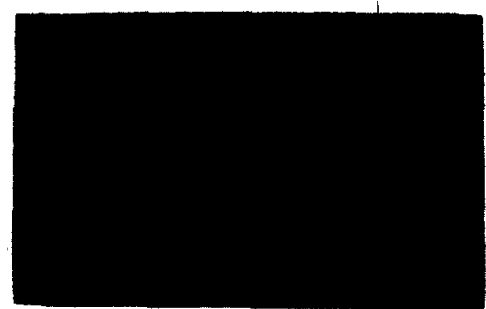

i i i

\section{(c) LARGE VORTEX, $d_{d} / \delta=3.1, \mathrm{~K} a_{v}=4.5$, METHANE}

Fig. 3. Quenching of a methane-air flame $(\phi=0.55)$ during an interaction with a vortex. Additional parameters appear in Tables 1 and 2. Each image represents $5 \mathrm{~cm}$ by $3 \mathrm{~cm}$. Colors represent the percentage of maximum superequilibrium OH fluorescence intensity: red $=50 \%-100 \%$; yellow $=30 \%-50 \%$; green $=20 \%-30 \%$; blue $=10 \%-20 \%$; and black $=0 \%-10 \%$. 

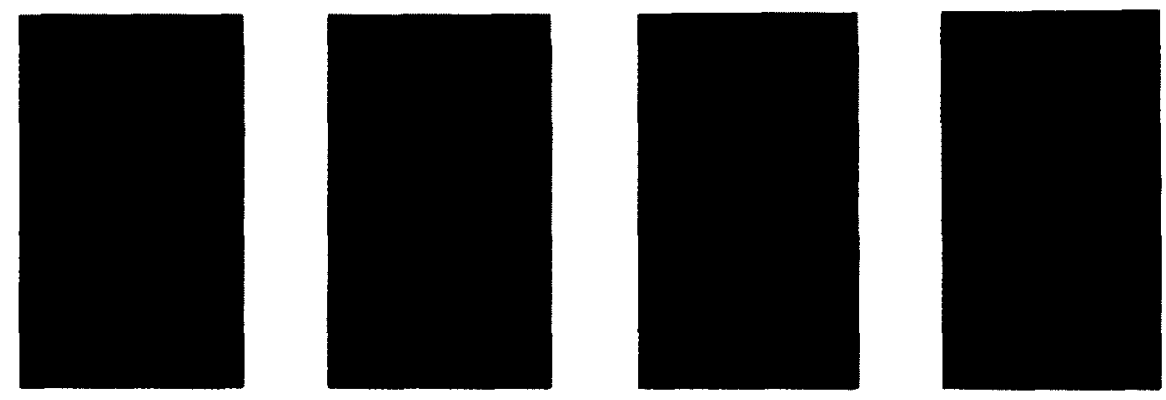

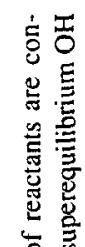
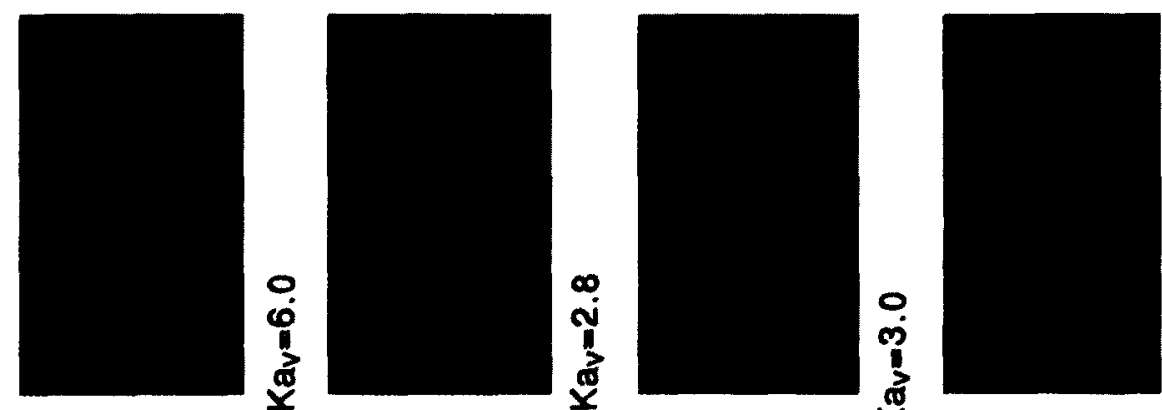

$\cong \Xi$

芩

욜

导

8,

홍.

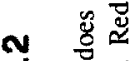

0 on

氞

离 $\bar{\Xi} \vec{~}$

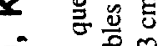

○. 동

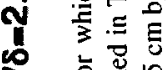

i
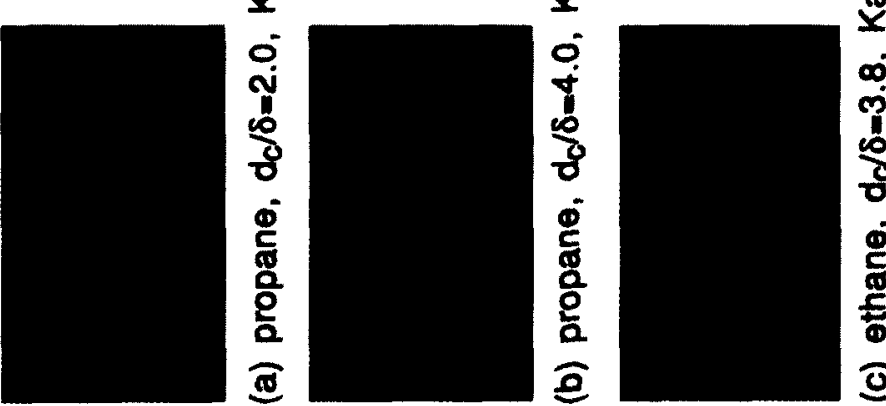

$0^{\circ}$

0 \%

$0^{-}$.

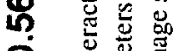

Q.

$\theta$ 可

Q

๘

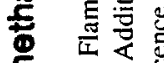

E

$\infty$ 常

อ

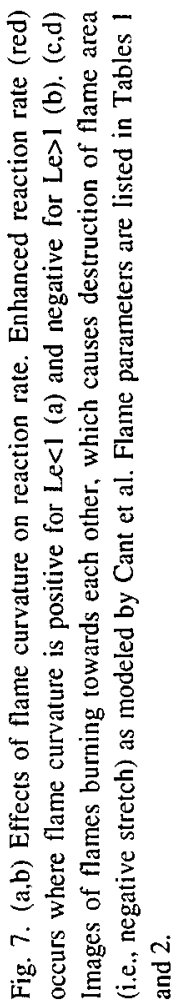


actants, as seen in Fig. 3, may be responsible for some of the unburned hydrocarbons and $\mathrm{CO}$ emitted by practical combustors. For the medium sized vortex, shown in Fig. $3 b$, there also is no roll-up of the flame into the vortex.

For the larger vorticies, Figs. $3 b$ and $3 c$ show that the flame rolls up about one revolution, which causes the pocket of reactants to be distorted into a thin, highly curved strip. Therefore, flame curvature effects can be just as pronounced, or even more pronounced for interactions involving large vortices than for interactions involving small vortices. That is, flame stretch in real interactions may not scale linearly with $U_{\theta} / d_{c}$ and thus the vortex Karlovitz number at quenching cannot be expected to have a single universal value. Flame burnthrough in the large vortex creates three separate pockets, which does not occur in the smaller vortex.

\section{Determination of the Thin Flame Limit on a Combustion Regime Diagram}

Two curves were determined from the $\mathrm{OH}$ images. The first is denoted the single vortex quenching curve and is shown in Fig. 4. This curve is then used to infer a second curve that is denoted the thin flame limit. To obtain the single vortex quenching curve shown in Fig. 4, vortices having six different core diameters

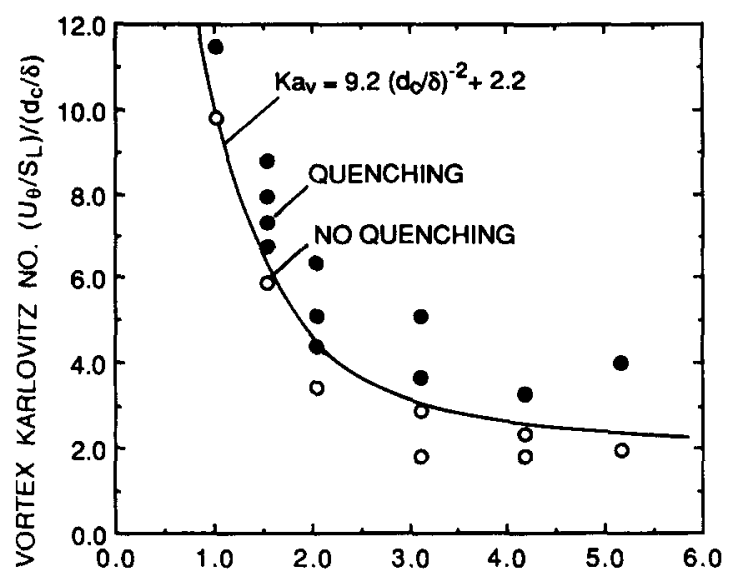

NONDIMENSIONAL VORTEX CORE DIAMETER do $\delta$

Fig. 4. Single vortex quenching curve. Methane-air flame, $\phi=0.55$. Quenching is determined from $\mathrm{OH}$ images such as Fig. 3. Solid curve represents best fit to the data. were chosen and $U_{\theta}$ was increased until local quenching was detected; i.e., the peak $\mathrm{OH}$ intensity of a segment is less than $1 \%$ of that of the undisturbed flame. The vortex Karlovitz numbers $\left(\mathrm{Ka}_{v}\right)$ that result in the quenching of the methane air flame $(\phi=0.55)$ are shown in Fig. 4, where $\mathrm{Ka}_{\mathrm{v}}$ is defined as $\left(U_{\theta} / S_{L}\right) /$ $\left(d_{c} / \delta\right)$. It is seen that small vortices are much less effective at quenching a flame (i.e., require larger Karlovitz numbers) than larger vortices, which has been predicted previously [4, 5]. Smaller vortices have smaller values of Reynolds number $\left(U_{\theta} D / \nu\right)$ and thus decay more rapidly as they enter the flame, where the kinematic viscosity is more than ten times larger than that of the unreacted gas. The single vortex quenching curve is represented by a Karlovitz number of:

$$
\left(U_{\theta} / S_{L}\right) /\left(d_{c} / \delta\right)=9.2\left(d_{c} / \delta\right)^{-2}+2.2
$$

for the methane-air flame at equivalence ratio of 0.55 . Other flames have different chemistry and radiative heat losses and may not be represented by Eq. 1 .

The single vortex quenching curve has been replotted in Fig. 5 in order to compare measurements to the numerical results of Poinsot et al. $[4,5]$. It is concluded that both curves display similar parabolic shapes. Absolute values differ because the simulation assumes single step chemistry as well as a radiative loss that differs from the experiment. In addition, the flame in the experiment experiences three-dimensional stretch and curvature whereas the simulations are two dimensional.

The thin flame limit is inferred from the present data using concepts discussed by Meneveau and Poinsot [24]. A turbulent flame is defined to exist in the thin flame regime if none of the eddies in its spectrum lie in the single vortex quenching region. A typical spectrum of the vorticies within a hypothetical turbulent flowfield is shown in Fig. 5. The rightmost point of the spectrum is defined by the rms velocity fluctuations $\left(u_{\mathrm{rms}}^{\prime}\right)$ and the integral scale $(L)$. The slope of the spectrum is onethird since $U_{\theta}$ scales as $u_{\mathrm{rms}}^{\prime}\left(d_{c} / L\right)^{1 / 3}$ in the turbulent cascade process [3]. To determine the thin flame limit, we seek values of $u_{\mathrm{rms}}^{\prime}$ and integral scale $L$ such that the spectrum in Fig. 


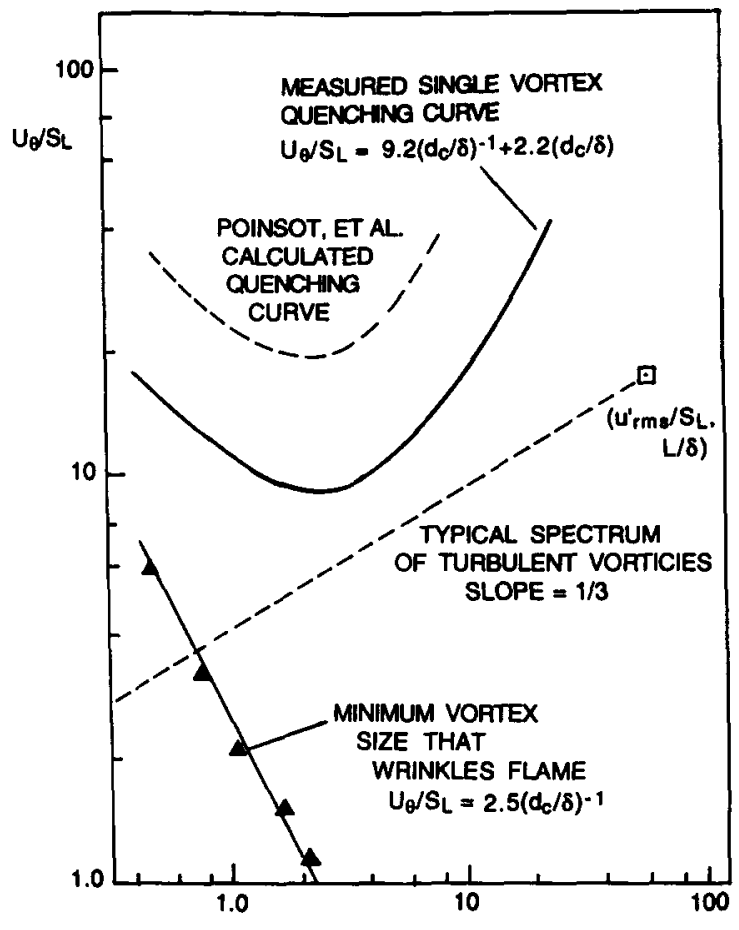

VORTEX CORE DIAMETER $\left(d_{c} / \delta\right)$

Fig. 5. The measured single-vortex quenching curve of Fig. 4 compared to the numerical results of Ref. 4. Methaneair, $\phi=0.55$. The measured minimum vortex size that wrinkles the flame was reported previously in Ref. 8.

5 lies below the measured single vortex quenching curve. Therefore a value of $L / \delta$ is chosen and a value of $u_{\mathrm{rms}}^{\prime} / S_{L}$ is chosen and a spectrum of vortices is plotted in Fig. 5 similar to that shown by the dashed line. The rectangular symbol in Fig. 5 represents chosen conditions of $u_{\mathrm{rms}}^{\prime} / S_{L}$ and $L / \delta$ equal to 16 and 61 , respectively. Holding $L / \delta$ constant, $u_{\mathrm{rms}}^{\prime} / S_{L}$ is then increased, causing the rectangular symbol to move upwards, until the dashed line that represents the spectrum first intersects the measured single vortex quenching curve. For this condition the values of $u_{\mathrm{rms}}^{\prime} / S_{L}$ and $L / \delta$ are plotted as one point on the thin flame limit of Fig. 6. The procedure is repeated for all other values of $L / \delta$, yielding the entire thin flame limit curve in Fig. 6, which is given by

$u_{\mathrm{rms}}^{\prime} / S_{L}=5.7(L / \delta)^{-1}+5.8(L / \delta)^{1 / 3}$

for the methane--air flame $(\phi=0.55)$. This thin flame limit is expected to depend on the fuel type and $\phi$ which control the chemical

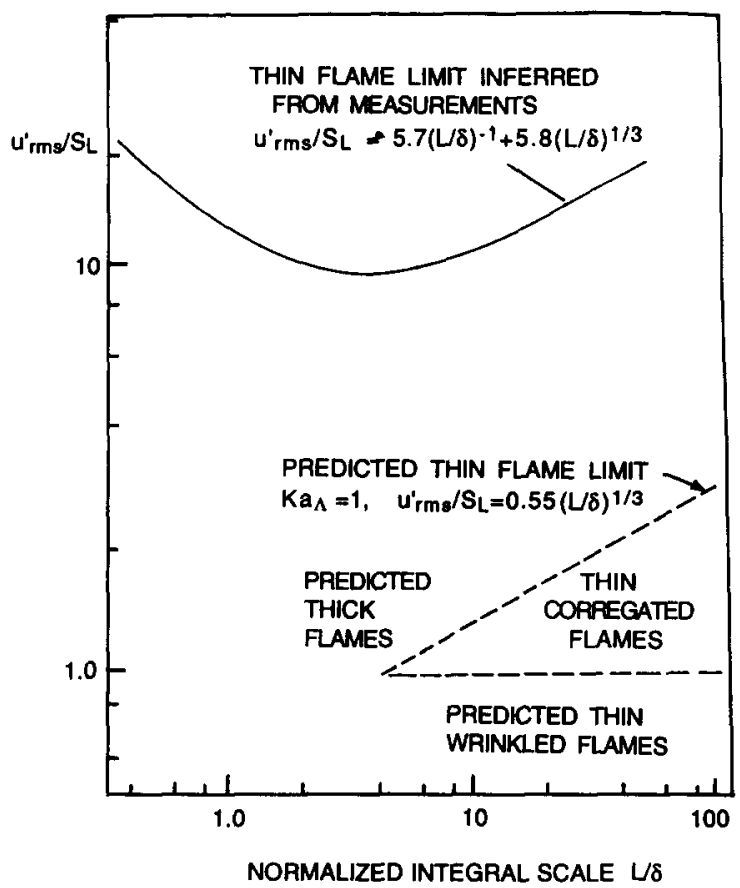

Fig. 6. The thin flame limit on a combustion regime diagram. Upper curve is inferred from the data shown in Fig. 4.

kinetic, molecular transport, and radiative losses.

Figure 6 represents the regime diagram for turbulent premixed flames. The inferred thin flame limit that is given by Eq. 2 is plotted in Fig. 6 and is compared to the Klimov-Williams criterion $\left(K a_{\Lambda}=1\right)$. The Taylor microscale [3] is defined to exactly equal $L\left(u_{\mathrm{rms}}^{\prime} L / \nu\right)^{-1 / 2}$; Prandtl number is 0.72 , and flame thermal thickness $\delta$ is $7.4 \alpha / S_{L}$ as discussed above. It is seen that the present measurements yield a thin flame limit in Fig. 6 that is an order of magnitude larger that the $K a_{\Lambda}=1$ criterion. The implication is that turbulent flames are expected to remain thin for significantly larger values of turbulence intensity than previously believed. A physical explanation is that the eddies at the Taylor microscale may characterize the maximum strain in nonreacting flows, but they are too small to survive the viscous forces within a flame.

The physical reasons why the thin flame limit in Fig. 6 has regions of negative and positive slopes are as follows. For large integral scale, the curve has positive slope, in agreement with the lower curve denoted the 
predicted thin flame limit because larger vorticies exert less strain on a flame than smaller vorticies, since strain scales as $U_{\theta} / d_{c}$. Thus as the integral scale is increases, an increased turbulence intensity is required for the vorticies to strain out and thicken the flame. However, as the integral scale is decreased sufficiently, the upper curve in Fig. 6 has a negative slope. The smaller vorticies are attenuated by viscous effects due to their small Reynolds number and significantly larger turbulence are required to change the flame structure.

\section{Turbulence Scales That Can Be Neglected in Simulations}

Measurements were made to determine the smallest vortex core diameter that wrinkles the flame, that is, causes a 5\% variation in the flame perimeter. Results are indicated by the lowermost curve in Fig. 5, which is given by

$$
U_{\theta} / S_{L}=2.5\left(d_{c} / \delta\right)^{-1} \text {. }
$$

Poinsot et al. $[4,5]$ define a cutoff scale as the value of $d_{c} / \delta$ for which the lowermost curve in Fig. 5 intersects the turbulence spectrum. A general relation for the cutoff scale for methane-air flames $(\phi=0.55)$ is deduced by combining Eq. 3 and the equation for the spectrum $\left(U_{\theta}=u_{\mathrm{rms}}^{\prime}\left(d_{c} / L\right)^{1 / 3}\right)$, which yields

$$
\left(d_{c} / \delta\right)_{\text {cutoff }}=2.0\left(u_{\mathrm{rms}}^{\prime} / S_{L}\right)^{-3 / 4}(L / \delta)^{1 / 4} \text {. }
$$

Vortex core diameters smaller than this cutoff scale have no effect on the flame in the present experiment. Therefore it is believed that such scales could be neglected from direct numerical simulations, discrete vortex simulations, or stochastic simulations of turbulent flames $[25,26]$. For typically turbulent flows the cutoff scale determined from Eq. 4 is substantially larger than the Kolmogorov or Taylor microscales. It is noted that lean methane-air flames are diffusionally unstable; we showed previously [8] that other fuels display significantly more diffusional stability and thus will have larger cutoff scales than a lean methane-air flame, so Eq. 4 is a conservative estimate of scales that can be neglected.

\section{Lewis Number Effects on Flame Curvature and Quenching}

The present images provide some of the first verification that flame curvature has a direct effect on the local reaction rate for conditions that simulate turbulent flames. Figure 7a illustrate the effects of flame curvature for a flame having Lewis number less than unity. At location 1 , where there is strong positive flame curvature, the reaction rate is enhanced and the region appears red. These results are in agreement with the analysis of Law [2], who shows how the reaction rate should increase where the local burned gas temperature is enhanced by the thermodiffusive effects. The local burned gas temperature $T_{b}$ is affected by flame stretch according to

$T_{b} / T_{\text {ad }}=1+\left(\mathrm{Le}^{-1}-1\right) \mathrm{Ka}$,

where $T_{\mathrm{ad}}$ is the adiabatic unstretched flame temperature [2]. At location 1 in Fig. 7a, the Karlovitz number is positive due to the positive flame curvature and $\left(\mathrm{Le}^{-1}-1\right)$ is positive. Therefore, Eq. 5 predicts that $T_{b}$ should be relatively large at location 1 , which is in accordance with the red region observed in Fig. 7a. Conversely, at location 2 the flame curvature is negative and the reaction rate (and the superequilibrium $\mathrm{OH}$ concentration, as indicated by the lower fluorescence intensity) are reduced, as denoted by the blue color. The opposite trends are observed in Fig. $7 \mathrm{~b}$ for a flame having Lewis number greater than unity. Positive flame curvature at location 3 causes a reduction in the reaction rate and the flame appears to be blue. Physically the heat flux vectors that are directed towards the reactants at location 3 are diverging. Negative flame curvature at location 4 enhances the reaction rate, as denoted by the red color. At location 4 the heat flux vectors are directed towards the reactants and they are focused, which enhances the reactant temperature. Thus all of the trends shown in Fig. 7 are in agreement with Eq. 5.

The present images also visualize the mechanism of flame sheet area reduction that has been predicted to occur in turbulent flames by Cant et al. [28] and Carrier et al. [29]. Figures 
$7 \mathrm{c}$ and $7 \mathrm{~d}$ show that a thin strip of reactants can exist between two flames that burn towards each other. The thin strip of reactants in Fig. 7 is a pocket that has been greatly elongated by positive stretch. As the two flames merge the total flame area rapidly decreases to zero in this region and the flame stretch ( $A^{-1} d A / d t$ ) must be negative. This mechanism of flame sheet destruction is believed to be present in turbulent flames and it prevents a flame from becoming infinitely wrinkled after long residence times in the turbulence field.

A similar set of experiments was run using both lean propane-air flames and lean ethane-air flames in order to study the effects of Lewis number on flame quenching. Figure 8a shows that a propane-air flame ( $\phi=0.52$, Le $=1.8$ ) does not quench for a Karlovitz number of 6.0. This Karlovitz number of 6.0 is larger than the value of 3.9 that is required to quench a methane-air flame for the same vortex size (Fig. 4); therefore it can be concluded that lean propane-air flames are more difficult to quench than lean methane-air flames. The theory of strained flames [2] predicts a trend that is opposite to that observed, namely that it should be easier to quench a lean propane-air flame (Le $>1$ ) with positive strain than a lean methane-air flame. The contradictory observations are believed to be due to complex chemistry at extinction that is not included in most theories.

Attempts were made to measure the quenching limit curves for lean propane-air and lean ethane-air flames, but quenching of such flames could not be achieved using the present experiment. Vortices became turbulent before quenching was observed if either $U_{\theta}$ or $d_{c}$ was increased. When $d_{c}$ was decreased in order to increase the Karlovitz number, quenching also was not observed because of the rapid decay of the curve in Fig. 4. Figures $8 \mathrm{a}-8 \mathrm{~d}$ show how it was deduced that quenching did not occur for various interactions. Pockets of reactants are observed to be entirely consumed for these cases.

\section{Measurements to Quantify Radiative Heat Losses}

The heat losses from the product gases were quantified by measuring the gas temperature on the combustor centerline as the flame passes over the TFP probe that was described earlier. Results are shown in Fig. 9. The maximum temperatures measured are 1\%-3\% less than the calculated adiabatic flame temperature for the five cases shown. The deviation is believed to be due to radiation losses from the product gases. Figure 9 shows that the temperature of the products decays in time by approximately $185 \mathrm{~K}$ during the first $200 \mathrm{~ms}$ after flame passage. To determine if radiative heat loss could be responsible for the entire observed temperature decay, the following calculation was performed. Gas at the same conditions as the products behind the methane-air flame $\left(\phi=0.60, T=1665 \mathrm{~K}, 6 \% \mathrm{CO}_{2}, 12 \% \mathrm{H}_{2} \mathrm{O}\right)$

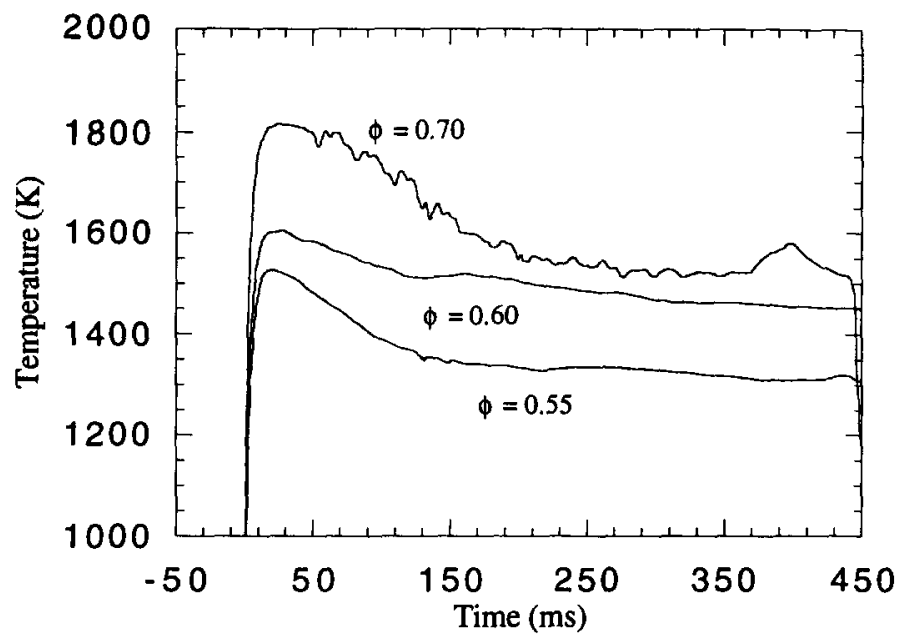

Fig. 9. Temperature time histories on centerline as the flame passes over the TFP filament for methane-air flames at various equivalence ratios. No vortex. 
was considered. The rate of temperature decay of the products due to radiative losses is predicted using

$\rho V c_{p} d T / d t=-\epsilon_{g} \sigma T^{4} A$,

where the surface-to-volume ratio $A / V$ of the chamber is $0.25 \mathrm{~cm}^{-1}$. The gas emissivity $\epsilon_{g}$ for the above partial pressures of $\mathrm{CO}_{2}$ an $\mathrm{H}_{2} \mathrm{O}$ and the chamber dimensions is 0.04 [30]. Integration of Eq. 6 yields product temperatures equal to $\left.T_{\text {ad }}{ }^{-3}+3 \sigma \epsilon_{g}\left((A / V) /\left(\rho c_{p}\right)\right) t\right)^{-1 / 3}$. The calculated temperature decrease at $200 \mathrm{~ms}$ is somewhat larger than the measured temperature decrease of $185 \mathrm{~K}$. Therefore it is concluded that radiative heat losses are sufficient to cause the measured temperature decay.

Figure 10 is a comparison of the measured temperature decay to that assumed for the numerical simulation of Poinsot et al. [4, 5]. The distance $x$ was computed for a given time after flame passage by using the measured velocity of the products in the laboratory coordinates. The distance that is required for the gas temperature to decay to $0.85 T_{\text {ad }}$ is found to be $30 \delta$, which is the three times larger than that calculated in the numerical simulations. Since Eq. 6 shows that heat loss is proportional to $d T / d t$, it is concluded that the heat loss assumed in the simulation is approximately three times larger than the realistic heat loss rate of the present experiment. It is not surprising that the propane-air flame of the numerical simulations was quenched by relatively weak vortices whereas the experimental pro- pane-air flames could not be quenched. Table 1 shows that the product gases must be cooled by radiation to approximately $1300 \mathrm{~K}$ before quenching could be observed, for the strain rates achieved in the present experiment. These results are in agreement with calculations of Darabiha et al. [10] who showed that quenching of methane-air premixed flames required that products be cooled to less than $1400 \mathrm{~K}$.

\section{CONCLUSIONS}

The physics of the quenching of a premixed flame by a vortex was studied using laser induced $\mathrm{OH}$ fluorescence imaging and thin filament pyrometry. By using a relatively thick laminar flame and small vortices, a ratio of the vortex core diameter to the thermal thickness of the flame was as small as unity.

1. Nondimensional vortex stretch rates $\left(\mathrm{Ka}_{v}\right)$ that characterize the size and strength of a vortex that quenches a premixed flame were found to increase as the vortex size decreases. Therefore, small vortices are significantly less efficient at quenching a flame than larger vortices.

2. The measured vortex quenching limit curve was:

$$
U_{\theta} / S_{L}=9.2\left(d_{c} / \delta\right)^{-1}+2.2\left(d_{c} / \delta\right)
$$

The measured curve displayed the same trends as predicted by direct numerical simulations, but absolute values differ because

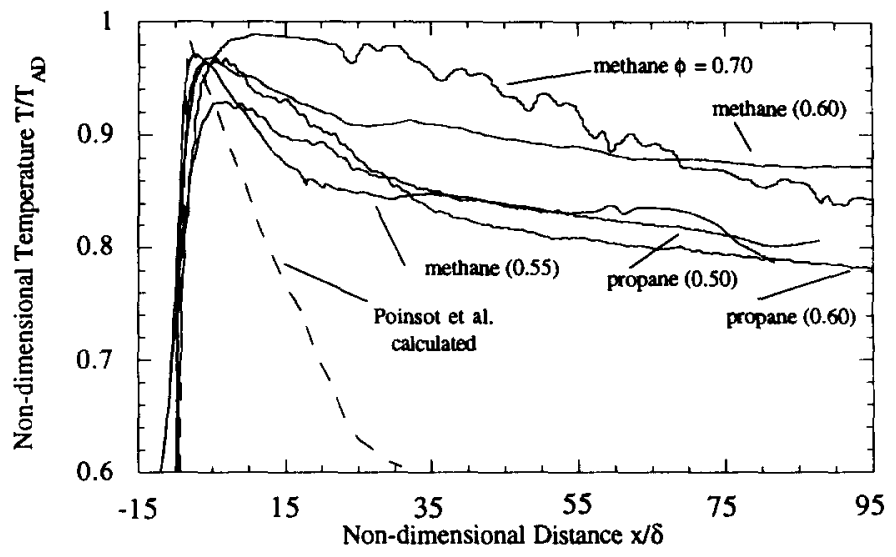

Fig. 10. Heat losses from the products, as characterized by TFP measurements of centerline temperature, compared with heat loss assumed in numerical simulations of Ref. 4 and 5. No vortex. Values of adiabatic flame temperature $T_{\text {ad }}$ and flame thickness $\delta$ are given in Table 2 . 
the simulation was two-dimensional, assumed unrealistically large heat losses, and used single-step chemical kinetics. The temperature of the products at which quenching was first detected was $1300 \mathrm{~K}$, which is in agreement with counterflow flame calculations with full chemistry.

3. The thin flame limit on the classical combustion regime diagram (Fig. 6) was inferred from the present data and the concepts proposed by Poinsot et al. to be

$u^{\prime} / S_{L}=5.7\left(d_{c} / \delta\right)^{-1}+5.8\left(d_{c} / \delta\right)^{1 / 3}$.

Results indicate that thin flames extend over a larger range of turbulence intensities than previously assumed.

4. It is inferred from the above experimental conclusion, combined with the theoretical work of Poinsot et al. that a significant fraction of the small scale turbulence can be neglected from direct simulations of flameturbulence interactions. It also follows that micromixing models that assume that small vortices play a dominant role are unrealistic.

5. Positive flame curvature can be observed to enhance the local reaction rate for Lewis number less than one, which is in agreement with the asymptotic analyses of Law and others.

6. Lean propane-air and ethane-air flames (having $\mathrm{Le}>1$ ) require significantly larger Karlovitz numbers in order to achieve quenching than lean methane-air flames (Le < 1); and Le $>1$ flames could not be quenched in the present apparatus. This observation disagrees with current stretched flame theory and may be attributed to complex chemistry effects.

The authors acknowledge useful discussions with Dr. Thierry Poinsot and Professor S. Candel during the stay of one of the authors (J. Driscoll) at Ecole Central de Paris. The effort of J.F.D. was partially supported by National Science Foundation Grant CTS 9123834. The cooperation of Dr. C. M. Roquemore made possible the TFP measurements; D. Trump and J. Ratcliffe assisted in the TFP and $\mathrm{OH}$ measurements.

\section{REFERENCES}

1. Williams, F. A., Combustion Theory, Addison Wesley Palo Alto, CA, 1989.

2. Williams, F. A., Combust. Flame 26:269-270 (1976).

3. Tennekes, H., and Lumley, J. L., A First Course in Turbulence, MIT Press, Cambridge, 1972.

4. Poinsot, T., Veynante, D., and Candel, S., TwentyThird Symposium (International) on Combustion, The Combustion Institute, Pittsburgh, 1990, pp. 613-619.

5. Poinsot, T., Veynante, D., and Candel, S., J. Fluia Mech. 228:561-606 (1991).

6. Magnussen, B. F., and Hjertager, B. H., Sixteenth Symposium (International) on Combustion, The Combustion Institute, Pittsburgh, 1977, p. 719.

7. Roberts, W. L., Driscoll, J. F., Drake, M. C., and Ratcliffe, J. W., Twenty-Fourth Symposium (International) on Combustion, The Combustion Institute, Pittsburgh, 1993, p. 169.

8. Roberts, W. L., and Driscoll, J. F., Combust. Flame 87:245-256 (1991).

9. Darabiha, N., Candel, S., and Marble, F. E., Combust. Flame 64:203-217 (1986).

10. Darabiha, N., Candel, S., Giovangigli, V., and Smooke, M. D., Combust. Sci. Technol. 60:267-272 (1988).

11. Ashurst, W. T., Sivashinsky, G. I., and Yakhot, V., Combust. Sci. Technol. 62:273-284 (1988).

12. Haworth, D. C., and Poinsot, T. J., J. Fluid Mech. 244:405-436 (1992).

13. Wu, M.-S., and Driscoll, J. F., Combust. Flame 91:310-322 (1992).

14. Warnatz, J., Eighteenth Symposium (International) on Combustion, The Combustion Institute, Pittsburgh, 1980, pp. 369-383.

15. Bechtel, J. H., Blint, R. J., Dasch, C. J., and Weinberger, D. A., Combust. Flame 42:197-213 (1981).

16. Friedman, R., Fourth Symposium (International) on Combustion, The Combustion Institute, Pittsburgh, 1952, p. 259.

17. Dixon-Lewis, G., and Williams, A., Eleventh Symposium (International) on Combustion, The Combustion Institute, Pittsburgh, 1966, p. 951.

18. Yamaoka, I., and Tsuji, H., Twentieth Symposium (Intemational) on Combustion, The Combustion Institute; Pittsburgh, 1984, pp. 1883-1892.

19. Yu, G. Law, C. K., and Wu, C. K., Combust. Flame 63:339-347 (1986).

20. Lewis, B., and von Elbe, G., Combustion, Flames, and Explosions of Gases, Academic, New York, 1961.

21. Drake, M. C., and Pitz, R. W., Exp. Fluids 3:283 (1985).

22. Goss, L. P., Vilimpoc, V., Sarka, B., and Lynn, W. F., Trans. ASME 111:46-52, (1989).

23. Drake, M. C., and Blint, R. J., Combust. Sci. Technol. 75:261 (1991).

24. Meneveau, C., and Poinsot, T., Combust. Flame 86:311-332 (1991).

25. Wu, M. S., Kwon, S., Driscoll, J. F., and Faeth, G. M., Combust. Sci. Technol. 83:187-202 (1992). 
26. Kwon, S., Wu, M. S., Driscoll, J. F., and Faeth, G. M., Combust. Flame 88:221-238 (1992).

27. Law, C. K., Twenty-Second Symposium (International) on Combustion, The Combustion Institute, Pittsburgh, 1988, pp. 1381-1402.

28. Cant, R. S., Pope, S. B., and Bray, K. N. C., TwentyThird Symposium (International) on Combustion, The Combustion Institute, Pittsburgh, 1990, pp. 809-815.
29. Carrier, G. F., Fendell, F. E., and Marble, F. E., SLAM J. Appl. Math 28:2,463 (1975).

30. Siegel, R., and Howell, J. R., Thermal Radiation Heat Transfer, 2nd ed., Hemisphere, New York, 1981, pp. 597-671.

Received 27 November 1992; revised 12 February 1993 